\author{
纳米材料在生物分析应用中存在的若干问题

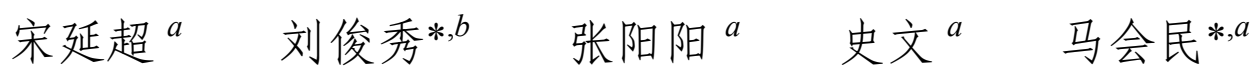 \\ ( ${ }^{a}$ 中国科学院化学研究所 活体分析化学重点实验室 北京 100190) \\ $\left({ }^{b}\right.$ 北京大学第三医院耳鼻咽喉科 北京 100191)
}

\begin{abstract}
摘要 从三个方面考察与总结了一些常用的纳米材料(如碲化镉量子点, 纳米金和碳纳米点)在生物分析应用中存在的 问题: (1)纳米材料的毒性. 三种裸露纳米材料的平行比较实验表明, 碲化镉量子点能够导致细胞代谢活性下降、细胞发 生皱缩、甚至死亡，具有很强的毒性; 纳米金在高浓度 $(30 \mu \mathrm{g} / \mathrm{mL})$ 时可对细胞代谢产生一定的抑制作用; 而碳纳米点对 细胞几乎不产生影响, 具有较好的生物相容性. 三种纳米材料的相对毒性为: 碲化镉量子点 $>>$ 纳米金 $>$ 碳纳米点. 这种相对毒性还得到了绿豆芽生长抑制实验的支持. (2)纳米材料的非均一性. 这主要表现在以下几个方面: 粒径分布 的非均一性, 表面修饰/性质的非均一性, 以及在生物样品(如细胞)中分布的非均一性. (3)纳米材料的环境敏感性或稳 定性. 实验表明, 碲化镉量子点、纳米金和碳纳米点的光学性质对环境 $\mathrm{pH}$ 的改变均十分敏感, 而且纳米金不抗盐, 在 离子强度较高的盐溶液中不稳定、易聚集. 这些问题的严重性在许多以往的研究中并未引起人们的全面重视. 我们希 望通过本研究以及对这些问题的再次探讨，能促使人们在实际应用中对相关纳米材料进行重新的审视和合理的选择. 此外, 为克服这些问题, 我们在文中提到的一些措施可供参考.

关键词＼cjkstart纳米材料; 碲化镉量子点; 纳米金; 碳纳米点; 毒性; 非均一性; 环境敏感性; 生物分析
\end{abstract}

\title{
Some Problems of Nanomaterials in Bioanalytical Applications
}

\author{
Song, Yanchao ${ }^{a} \quad$ Liu, Junxiu* ${ }^{*, b}$ Zhang, Yangyang $^{a} \quad$ Shi, Wen ${ }^{a} \quad$ Ma, Huimin*,a \\ ( ${ }^{a}$ Beijing National Laboratory for Molecular Sciences, Key Laboratory of Analytical Chemistry for Living Biosystems, \\ Institute of Chemistry, Chinese Academy of Sciences, Beijing 100190) \\ ( ${ }^{b}$ Department of Otorhinolaryngology, Peking University Third Hospital, Beijing 100191)
}

\begin{abstract}
By taking the three commonly used CdTe quantum dots (CdTe QDs), Au nanoparticles (Au NPs) and carbon nanodots (C-dots) as example, we have demonstrated and summarized some noteworthy problems of nanomaterials in bioanalytical applications. These problems mainly include the toxicity, microscopic heterogeneity and environmental susceptivity of nanomaterials, which had been separately discussed with different depths but unfortunately are often overlooked in practice. In the present work, a parallel comparative investigation was first made on cytotoxicity, which reveals that CdTe QDs can lead to the decrease of cell metabolic activity, cell shrinkage, and even cell death; Au NPs at a higher concentration $(30 \mu \mathrm{g} / \mathrm{mL})$ decrease the metabolic activity of cells to a small extent; C-dots exert no obvious toxicity to live cells, showing good biocompatibility. These studies indicate that the relative toxicity of the three nanomaterials in their bare forms is CdTe QDs $>>$ AuNPs $>$ C-dots, which is further supported by the inhibition experiment on the growth of green gram sprouts. Second, the microscopic heterogeneity of nanomaterials was compared with that of resorufin (a small molecular fluorescent dye) by cell imaging experiments, which clearly shows that the distribution of nanomaterials in cells is much less uniform than that of small molecular probes. Besides, the heterogeneity of both size distribution and surface modification/property of nanomaterials is also unavoidable. Third, the environmental susceptivity of nanomaterials was studied, and the results showed that spectroscopic properties of the above three nanomaterials are all sensitive to the $\mathrm{pH}$ change of solution. Moreover, AuNPs are also rather susceptible to the ionic strength of solution. The above three problems should arouse high attention of researchers in some bioanalyses. In addition, the measures that we suggested in the paper may be helpful to solve these problems to some extent.
\end{abstract}

Keywords nanomaterials; CdTe quantum dots; Au nanoparticles; carbon nanodots; toxicity; microscopic heterogeneity; environmental susceptivity; bioanalysis

\footnotetext{
*E-mail: mahm@iccas.ac.cn; liujunxiusanyuan@sina.com

Received August 29, 2013; published September 10, 2013

Supporting information for this article is available free of charge via the Internet at http://sioc-journal.cn.

Project supported by the National Natural Science Foundation of China (No. 21321003), the 973 Program (2011CB935800, and 2010CB933502) and the Chinese Academy of Sciences.

项目受国家自然科学基金(No. 21321003)、973 计划(2011CB935800, 2010CB933502)和中国科学院资助.
} 


\section{1 引言}

一些纳米材料(如碲化镉量子点, 纳米金和碳纳米 点等)因具有独特的光学性质, 目前已广泛用于各个领 域，如细胞荧光成像、分析检测、药物释放及治疗 等 ${ }^{[1 \sim 10]}$. 然而, 由于纳米材料本身固有的特性以及使用 环境的复杂性, 它们在实际生物分析应用中所暴露的诸 如毒性、非均一性、环境敏感性等若干问题则越来越令 人担忧. 虽然纳米材料的毒性和环境敏感性已有较深入 的研究 ${ }^{[3,11,12]}$, 且其非均一性在相关的工作中也有所提 及 ${ }^{[13]}$, 但是这些问题在实际应用中的严重性尚未引起 人们的高度重视. 在此, 我们以目前常用的三种纳米材 料碲化镉量子点(CdTe QDs)、纳米金(AuNPs)和碳纳米 点(C-dots)为例, 通过具体地考察它们在实际生物分析 应用中的行为, 较全面地揭示了其存在的毒性、非均一 性和环境敏感性等问题. 我们希望该研究能促使人们对 相关纳米材料进行重新的审视, 以便从实际应用角度出 发合理地加以选择与使用.

具体地讲, 我们首先按照文献 ${ }^{[12]}$ 并采用水热法、柠 檬酸钠还原法和微波裂解法分别制备与表征了 $\mathrm{CdTe}$ QDs, AuNPs 和 C-dots 三种裸露纳米材料. 在此基础上, 通过考察它们的细胞毒性、植物毒性以及受介质的 $\mathrm{pH}$ 和离子强度等环境因素的影响, 并结合细胞荧光成像分 析, 归纳与总结了这些纳米材料在实际生物分析应用中 所存在的毒性、非均一性以及环境敏感性等弊端, 并希 望人们对这些问题给予充分的重视.

\section{2 结果与讨论}

\section{1 纳米材料的毒性}

首先, 纳米材料的毒性不容忽视. 虽然借助各种表 面修饰技术可降低纳米材料的毒性，但事实上它们不可 避免地会有一部分残留在使用的场所(如细胞内), 并最 终降解为裸露的形式 ${ }^{[12,14,15]}$. 因此, 考察未经修饰的裸 露结构的纳米材料才能客观反映出其真实的毒性. 图 1 示出了用四甲基偶氮唑盐比色法(MTT 方法)得到的细 胞毒性实验结果. 由此图可以看出, 高于 $1 \mu \mathrm{g} / \mathrm{mL}$ 的 CdTe QDs 即可导致细胞活性丧失 $30 \%$ 以上, Au NPs 的 浓度在高达 $30 \mu \mathrm{g} / \mathrm{mL}$ 时仅引起约 $10 \%$ 的活性降低, 而 C-dots 在相同条件下尚未显示明显的毒性. 这表明, 三 种纳米材料的相对毒性是: CdTe QDs $>>\mathrm{Au}$ NPs $>$ C-dots.

图 2 是 HeLa 细胞经不同浓度的纳米材料处理后得 到的微分干涉相差成像情况. 由此图可以看出, 10 $\mu \mathrm{g} / \mathrm{mL}$ 的 CdTe QDs 即可使 HeLa 细胞发生皱缩, 50 $\mu \mathrm{g} / \mathrm{mL}$ 的 CdTe QDs 则可导致 HeLa 细胞死亡而不再贴 壁; 在相同的实验条件下, Au NPs 和 C-dots 对细胞的形 貌几乎没有产生影响. 此外, 对植物豆芽的生长抑制实
验也证实了这三种纳米材料的上述相对毒性，且以 CdTe QDs 的毒性为最大(图 S1, 支持信息). 这些结果不 仅与我们先前的发现一致 ${ }^{[12]}$, 而且也与其他研究人员 的观察吻合 ${ }^{[11,16,17]}$. 值得指出的是，尽管在本实验的短 时间内 C-dots 没有显现毒性，但也不能排除在长时间内 产生毒副作用. 因此，鉴于纳米材料、特别是 CdTe QDs 的高毒性, 应尽量避免将其应用于生物活体与药物输送 (相对而言, 这些材料较适用于生物体外的分析检测).

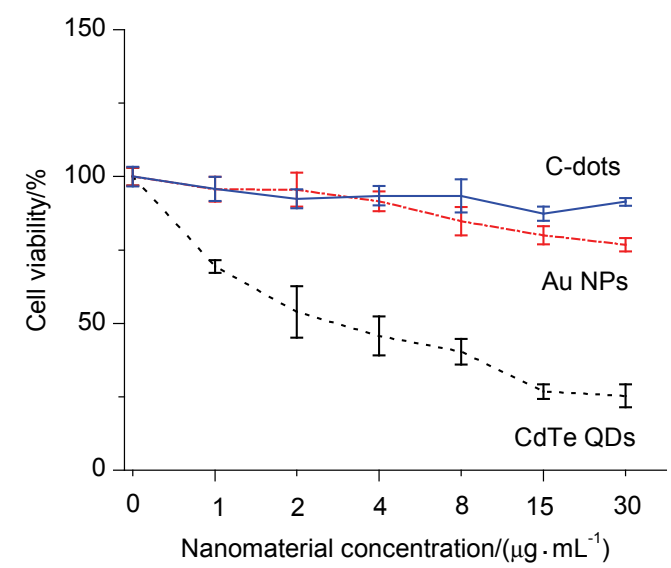

图 1 细胞毒性实验. $\mathrm{HeLa}$ 细胞与不同浓度的纳米材料于 $37{ }^{\circ} \mathrm{C}$ 捊育 $24 \mathrm{~h}$ 后其代谢活性的变化 (未经纳米材料处理的 HeLa 细胞作对照组, 其活性设定为 $100 \%$; 实验重复 5 次). 实验参照文献 ${ }^{[12]}$ 操作

Figure 1 Viability changes of HeLa cells after $24 \mathrm{~h}$ incubation with CdTe QDs, Au NPs, and C-dots at varied concentrations (the viability of the cells in the absence of the nanomaterial is defined as $100 \%$ ). The results were the mean \pm SD of 5 separate measurements. The experiments were made as described previously ${ }^{[12]}$

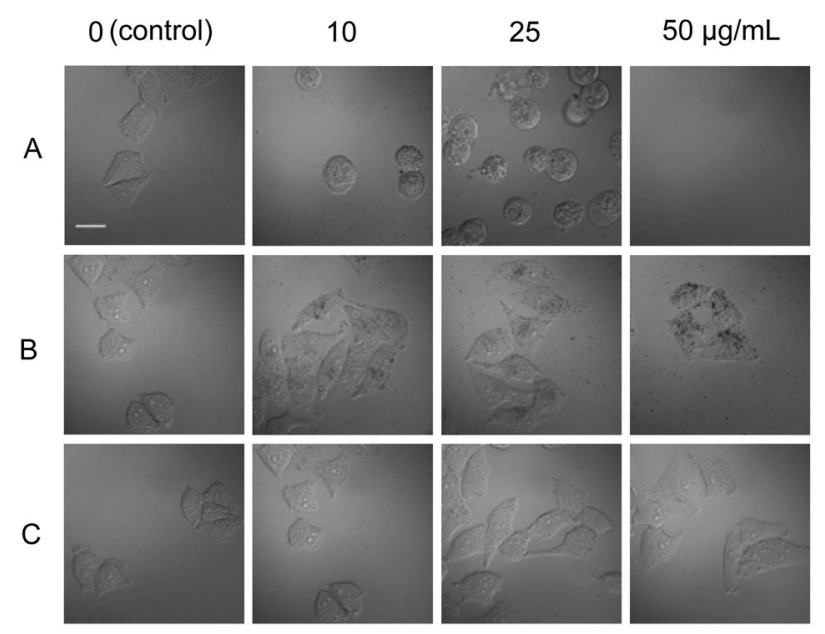

图 $2 \mathrm{HeLa}$ 细胞经不同浓度 $(0,10,25,50 \mu \mathrm{g} / \mathrm{mL})$ 的纳米材料(A: CdTe QDs; B: AuNPs; C: C-dots)于 $37{ }^{\circ} \mathrm{C}$ 捊育 $24 \mathrm{~h}$ 后得到的微分干涉相差 (DIC)成像图. 标尺, $10 \mu \mathrm{m}$. 实验参照文献 ${ }^{[12]}$ 操作

Figure 2 The differential interference contrast (DIC) images of HeLa cells treated at $37{ }^{\circ} \mathrm{C}$ for $24 \mathrm{~h}$ with different nanomaterials (A: CdTe QDs; B: AuNPs; C: C-dots) at various concentrations $(0,10,25$, and 50 $\mu \mathrm{g} / \mathrm{mL}$ ). Scale bar, $10 \mu \mathrm{m}$. The experiments were made as described previously ${ }^{[12]}$ 


\section{2 纳米材料的非均一性}

其次, 纳米材料的非均一性主要表现在三个方面: (1)粒径分布的非均一性. 通常, 制备的纳米材料的粒径 不是均一的固定数值, 而呈正态分布. 例如, 本工作中 制备与使用的 CdTe QDs, Au NPs 以及 C-dots 颗粒的水 合直径均值分别为 $3.9,22$ 和 $3.0 \mathrm{~nm}$, 但其直径的分布范 围却可分别达 $2.5 \sim 6.5,14 \sim 51$ 和 $2 \sim 6.5 \mathrm{~nm}$. (2)表面修 饰的非均一性. 在实际应用中, 如果需要对纳米材料进 行表面修饰，则操作者很难保证修饰基团的分布完全均 匀. 这种表面基团修饰的非均一性，连同颗粒直径分布 的非均一性, 将会导致纳米颗粒的性质甚至实验结果的 非重现性. (3)在生物样品中分布的非均一性. 与有机小 分子苂光染料相比, 纳米材料在生物样品(如细胞)中的 分散性通常较差. 这一点很少有人提及并进行对比研 究 ${ }^{[13]}$. 在此, 我们借助激光共聚焦荧光成像方法, 比较 了小分子荧光染料试卤灵(resorufin)与 3,4-二硝基苯甲 酰胺功能化的 $\mathrm{CdTe} / \mathrm{ZnTe} \mathrm{QDs}^{[18]}$ 以及叶酸功能化的 C-dots ${ }^{[19]}$ 在 HeLa 细胞中的分布行为. 如图 3 所示, $\mathrm{CdTe} / \mathrm{ZnTe}$ QDs 和 C-dots 在 HeLa 细胞的不同区域中呈 现出的荧光亮度存在着显著差异(图 3A 和图 3B), 特别 是在负载 C-dots 的细胞中表现出不规则的斑点(图 3B). 这清楚地说明, 纳米材料在细胞中的分布非常不均匀. 相比之下, 负载有机染料的细胞其荧光亮度无论是在细 胞核还是在细胞质中都非常均匀(图 3C). 已报道的一些 电镜实验结果也显示纳米材料(如 $\mathrm{Au} \mathrm{NPs}$ )在细胞中的 分布是不均匀的 ${ }^{[16,20]}$. 纳米材料的非均一性可能决定着 实验结果的重复性. 这一点应引起人们的重视. 或许, 发展粒径单一或分布较窄的纳米材料的制备新方法、以
A
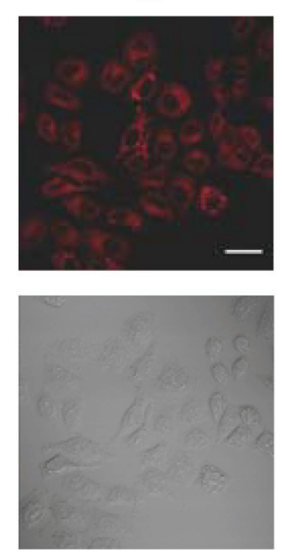

C
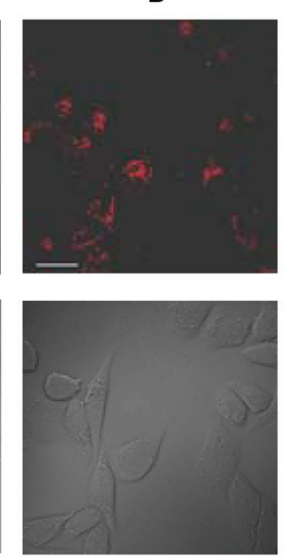
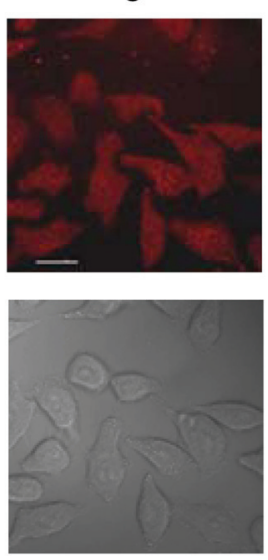

图 $3 \mathrm{HeLa}$ 细胞分别与(A) CdTe/ZnTe QDs $(30 \mu \mathrm{g} / \mathrm{mL}) 、$ (B) C-dots (50 $\mu \mathrm{g} / \mathrm{mL})$ 和 $(\mathrm{C})$ resorufin $\left(10 \mu \mathrm{mol} \cdot \mathrm{L}^{-1}\right)$ 于 $37{ }^{\circ} \mathrm{C}$ 孵育 2,6 和 $0.5 \mathrm{~h}$ 后的苂 光成像图. 标尺, $20 \mu \mathrm{m}$. 苂光成像实验参照文献 ${ }^{[18]}$ 操作

Figure 3 Fluorescence images of HeLa cells after incubated with (A) CdTe/ZnTe QDs (30 $\mu \mathrm{g} / \mathrm{mL})$, (B) C-dots $(50 \mu \mathrm{g} / \mathrm{mL})$, and (C) resorufin $\left(10 \mu \mathrm{mol} \cdot \mathrm{L}^{-1}\right)$ at $37{ }^{\circ} \mathrm{C}$ for 2,6 , and $0.5 \mathrm{~h}$, respectively. Scale bars, 20 $\mu \mathrm{m}$. Fluorescence imaging experiments were made as described previously ${ }^{[18]}$
及选用粒径尽可能小的纳米颗粒 ${ }^{[13]}$, 可在一定程度上 减小其非均一性问题.

\section{3 纳米材料的环境敏感性}

最后, 我们还考察了溶液环境(如 $\mathrm{pH}$, 离子强度)对 CdTe QD, AuNPs 和 C-dots 的光学性质的影响. 结果发 现, 利用水热法制备的 CdTe QDs 对 $\mathrm{pH}$ 的敏感现象不会 随着稳定剂种类 [颈基丙酸(MPA), 谷胱甘肽(GSH)或半 胱氨酸 $(L-\mathrm{cys})]$ 的不同而改变(图 S2, 支持信息). 图 4 示 出了以稳定剂 MPA 为例所合成的 CdTe QDs 的荧光随 溶液 $\mathrm{pH}$ 的变化情况. 由此图可以看出, CdTe QDs 在碱 性介质中具有较强的荧光，但当 $\mathrm{pH}$ 低于 5 时，其荧光几 乎被完全淬灭. 这与其他研究者观察到的现象一致 ${ }^{[21,22]}$. 然而, 值得注意的是, 即使拥有核壳结构的 $\mathrm{CdTe} / \mathrm{ZnTe}$ QDs 也表现出 $\mathrm{pH}$ 的敏感性(图 S3, 支持信息). 此外, 研 究表明, Au NPs 与 C-dots 的光学性质也随着 $\mathrm{pH}$ 的改变 而改变(图 S4, S5, 支持信息). 这些纳米材料的 $\mathrm{pH}$ 敏感 性会对一些分析结果的准确度造成严重影响.

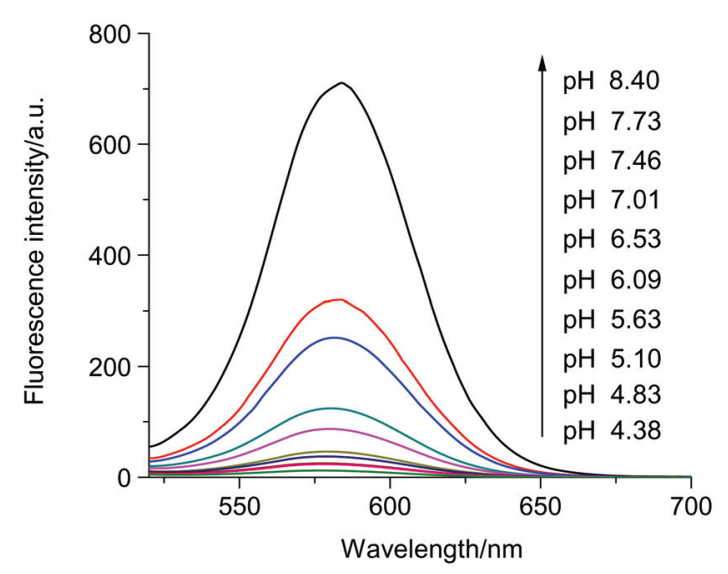

图 4 以 MPA 为稳定剂合成的 CdTe QDs $(60 \mu \mathrm{g} / \mathrm{mL})$ 在不同 $\mathrm{pH}$ 水溶 液中(用稀 $\mathrm{NaOH}$ 或 $\mathrm{HCl}$ 调 $\mathrm{pH}$ ) 的苂光光谱. $\lambda_{\mathrm{ex}}=500 \mathrm{~nm}$

Figure 4 Fluorescence spectra of $60 \mu \mathrm{g} / \mathrm{mL}$ CdTe QDs (prepared with MPA as ligand) in different $\mathrm{pH}$ solutions adjusted by diluted $\mathrm{NaOH}$ or $\mathrm{HCl} . \lambda_{\mathrm{ex}}=500 \mathrm{~nm}$

离子强度的影响实验表明, CdTe QDs 和 C-dots 的苂 光光谱在不同离子强度的溶液中可保持相对稳定(图 S6, S7, 支持信息). 然而, 对于 $\mathrm{Au} \mathrm{NPs}$ 而言, 离子强度的影 响却颇为显著. 如图 5 所示, 随着溶液中 $\mathrm{NaCl}$ 浓度的增 加, $\mathrm{Au} \mathrm{NPs}$ 由酒红色逐渐变为蓝色, 表明 $\mathrm{Au}$ NPs 在 $\mathrm{NaCl}$ 等盐溶液中极易聚集(分散性良好的 $\mathrm{Au} N \mathrm{NP}$ 溶液 通常呈酒红色，而聚集的 $\mathrm{Au} \mathrm{NPs}$ 呈蓝色 ${ }^{[23]}$ ). 正由于这 一弊端，许多已报道的基于 $\mathrm{Au}$ NPs 的检测体系都没有 使用缓冲剂. 显然, 在无缓冲剂存在的情况下, 溶液 $\mathrm{pH}$ 的小变动将会对分析结果造成影响.上述研究表明，利 用纳米光学传感体系进行分析检测时，应严格控制溶液 的环境因素(如 $\mathrm{pH}$ 、离子强度、甚至温度等)的改变, 以 尽量减少其影响. 


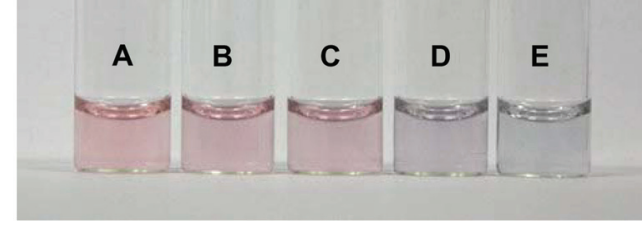

图 5 纳米金 $\left(6 \mathrm{nmol} \cdot \mathrm{L}^{-1}\right)$ 在不同浓度 $\mathrm{NaCl}\left(\mathrm{A}: 0 \mathrm{mmol} \cdot \mathrm{L}^{-1} ; \mathrm{B}: 10\right.$ $\mathrm{mmol} \cdot \mathrm{L}^{-1}$; $\mathrm{C}: 25 \mathrm{mmol} \cdot \mathrm{L}^{-1}$; D: $50 \mathrm{mmol} \cdot \mathrm{L}^{-1}$; $: 100 \mathrm{mmol} \cdot \mathrm{L}^{-1}$ ) 溶液中 的颜色变化

Figure 5 Color change of AuNPs $\left(6 \mathrm{nmol} \cdot \mathrm{L}^{-1}\right)$ in aqueous solution with various $\mathrm{NaCl}$ concentrations $\left(\mathrm{A}: 0 \mathrm{mmol} \cdot \mathrm{L}^{-1}\right.$; $\mathrm{B}: 10 \mathrm{mmol} \cdot \mathrm{L}^{-1}$; $\mathrm{C}: 25$ $\mathrm{mmol} \cdot \mathrm{L}^{-1}$; D: $50 \mathrm{mmol} \cdot \mathrm{L}^{-1}$; E: $100 \mathrm{mmol} \cdot \mathrm{L}^{-1}$ )

\section{3 结论}

综上所述, 我们以一些常用的纳米材料(CdTe QDs, $\mathrm{Au}$ NPs 和 C-dots)为例, 从毒性、非均一性和环境敏感 性这三方面考察并归纳了其在实际生物分析应用中存 在的问题. 这些问题的严重性在许多以往的研究中并未 引起人们的全面重视. 我们希望通过本研究以及这些问 题的再次提出, 能促使人们在实际应用中对相关纳米材 料进行重新的审视和合理的选择.

\section{References}

[1] Shi, W.; Li, X. H.; Ma, H. M. Angew. Chem., Int. Ed. 2012, 51, 6432.

[2] Bhang, S. H.; Won, N.; Lee, T. J.; Jin, H.; Nam, J.; Park, J.; Chung, H.; Park, H. S.; Sung, Y. E.; Hahn, S. K.; Kim, B. S.; Kim, S. ACS Nano 2009, 3, 1389.

[3] Lee, H.; Lee, K.; Kim, I. K.; Park, T. G. Biomaterials 2008, 29, 4709.

[4] Zhu, A. W.; Qu, Q.; Shao, X. L.; Kong, B.; Tian, Y. Angew. Chem., Int. Ed. 2012, 51, 7185.

[5] Zhu, J.; Liao, L.; Zhu, L. N.; Kong, J. L.; Liu, B. H. Acta Chim. Sinica 2013, 71, 69. (朱杰, 廖蕾, 朱丽娜, 孔继烈, 刘宝红, 化学
学报, 2013, 71, 69.)

[6] Luo, J. S.; Wang, Y. Y.; Tan, K. J. Acta Chim. Sinica 2012, 70, 1945. (罗金尚, 王莹莹, 谭克俊, 化学学报, 2012, 70, 1945.)

[7] Men, J. Y.; Gao, B. J.; Chen, Z. P.; Yao, L. Acta Chim. Sinica 2012 , 70, 2273. (门吉英, 高保娇, 陈志萍, 么兰, 化学学报, 2012, 70, 2273.)

[8] Wen, Q. S.; Tang, H. W.; Yang, G. M.; Liu, L. B.; Lv, F. T.; Yang, Q.; Wang, S. Acta Chim. Sinica 2012, 70, 2137. (温泉山, 唐红伟, 杨高买，刘礼兵，吕风婷，杨琼，王树，化学学报，2012，70, 2137.)

[9] Zhang, Z. M.; Liu, R.; Xu, D. M.; Liu, J. F. Acta Chim. Sinica 2012 70, 1686. (张宗绵, 刘睿, 徐敦明, 刘景富, 化学学报, 2012, 70, 1686.)

[10] Wang, J. Y.; Song, D. D.; Bao, Y. M. Acta Chim. Sinica 2012, 70, 1193. (王静云，宋丹丹，包永明，化学学报, 2012, 70, 1193.)

[11] Jasmina, L.; Sung, J. C.; Francoise, M. W.; Dusica, M. Chem. Biol. 2005, 12, 1227.

[12] Song, Y. C.; Feng, D.; Shi, W.; Li, X. H.; Ma, H. M. Talanta 2013 116, 237.

[13] Feng, D.; Song, Y. C.; Shi, W.; Li, X. H.; Ma, H. M. Anal. Chem. 2013, 85,6530 .

[14] Derfus, A. M.; Chan, W. C. W.; Bhatia, S. N. Nano Lett. 2004, 4, 11.

[15] Kirchner, C.; Liedl, T.; Kudera, S.; Pellegrino, T.; Javier, A. M.; Gaub, H. E.; Stollzle, S.; Fertig, N.; Parak, W. J. Nano Lett. 2005, 5, 331.

[16] Soenen, S. J.; Manshian, B.; Montenegro, J. M.; Amin, F.; Meermann, B.; Thiron, T.; Cornelissen, M.; Vanhaecke, F.; Doak, S. Parak, W. J.; Smedt, S. D.; Braeckmans, K. ACS Nano 2012, 6 , 5767.

[17] Yang, S. T.; Wang, X.; Wang, H. F.; Lu, F. S.; Luo, P. G.; Cao, L.; Meziani, M. J.; Liu, J. H.; Liu, Y. F.; Chen, M.; Huang, Y. P.; Sun, Y. P. J. Phys. Chem. C 2009, 113, 18110.

[18] Lu, J. X.; Song, Y. C.; Shi, W.; Li, X. H.; Ma, H. M. Chin. J. Chem. 2013, 31, 472 .

[19] Song, Y. C.; Shi, W.; Chen, W.; Li, X. H.; Ma, H. M. J. Mater Chem. 2012, 22, 12568

[20] Choi, S. Y.; Jeong, S.; Jang, S. H.; Park, J.; Park, J. H.; Ock, K. S.; Lee, S. Y.; Joo, S. W. Toxicol. In Vitro 2012, 26, 229.

[21] Sui, C. X.; Liu, Y. F.; Li, P. A.; Zhang, D.; Xia, F. Anal. Methods 2013, 5, 1695 .

[22] Mandal, A.; Tamai, N. J. Phys. Chem. C 2008, 112, 8244.

[23] Feng, D.; Zhang, Y. Y.; Shi, W.; Li, X. H.; Ma, H. M. Chem. Commun. 2010, 46, 9203 . 